MATHEMATICS OF COMPUTATION

Volume 76, Number 257, January 2007, Pages 137-152

S $0025-5718(06) 01897-7$

Article electronically published on August 31, 2006

\title{
A GENERALIZED BPX MULTIGRID FRAMEWORK COVERING NONNESTED V-CYCLE METHODS
}

\author{
HUO-YUAN DUAN, SHAO-QIN GAO, ROGER C. E. TAN, AND SHANGYOU ZHANG
}

\begin{abstract}
More than a decade ago, Bramble, Pasciak and Xu developed a framework in analyzing the multigrid methods with nonnested spaces or noninherited quadratic forms. It was subsequently known as the BPX multigrid framework, which was widely used in the analysis of multigrid and domain decomposition methods. However, the framework has an apparent limit in the analysis of nonnested V-cycle methods, and it produces a variable V-cycle, or nonuniform convergence rate $\mathrm{V}$-cycle methods, or other nonoptimal results in analysis thus far.

This paper completes a long-time effort in extending the BPX multigrid framework so that it truly covers the nonnested V-cycle. We will apply the extended BPX framework to the analysis of many V-cycle nonnested multigrid methods. Some of them were proven previously only for two-level and W-cycle iterations. Some numerical results are presented to support the theoretical analysis of this paper.
\end{abstract}

\section{INTRODUCTION}

The multigrid method, consisting of the fine-level smoothing and the coarse-level correction, is an effective iterative method for solving the linear system arising from, e.g., the finite element discretization of boundary-value problems. The multigrid method provides the optimal-order computation in such a case, in the sense that the number of arithmetic operations is proportional to the number of unknowns in the system of linear equations; cf. [1, [5], 28, 31, 33. The constant rate of $\mathrm{W}$-cycle multigrid iterations was proved in several early papers, one of them is [1, which is generalized to many nonnested cases, for example, [13, [15, 42, 43].

The multigrid method is often nonnested because the multilevel discrete spaces may not be nested, or discrete bilinear forms may be different on different levels. For example, the nonnestedness may be caused by bubble elements [4], composite elements [18, nonconforming elements 4, 13, nonnested meshes 42, the mortar method [2], 25], numerical integrations [24, or other situations (cf. [11]), such as finite difference equations. The multigrid methods with noninherited forms but nested spaces, other than the cases in [11, are studied in [26, 27, 30 for the discontinuous Galerkin method and the edge element. Many earlier two-level and $\mathrm{W}$-cycle nonnested multigrid iterations were analyzed by extending the method of 11. However, a generalized framework [11, referred as the BPX multigrid framework, is widely used in the analysis of multigrid iterations; e.g., [5], 6], 8], 9], 10],

Received by the editor July 21, 2001 and, in revised form, November 27, 2005.

2000 Mathematics Subject Classification. Primary 65N55, 65N30, 65F10.

Key words and phrases. V-cycle nonnested multigrid method. 
[12, 23], 25], 27], 32, 34], 39], 40], 37]. The framework is rooted in [7] and 38 .

Although the convergence theory for the $\mathrm{W}$-cycle was established [1, 11, 15], [4], the problem of how to establish the convergence rate for the $\mathrm{V}$-cycle nonnested multigrid method is subtle, and is still an active research subject; see [3, 11, [10, 9], 7], 16, 28, 20], 21], 29, 6], 12], etc. The BPX framework [11] was generalized to allow nonsymmetric smoothings and can be applied to some nonnested multigrid methods. In particular, it provides a constant convergence rate for the nonnested $\mathrm{V}$-cycle under the assumption that

$$
A_{k}\left(I_{k} u, I_{k} u\right) \leq A_{k-1}(u, u) \quad \forall u \in U_{k-1}, \forall k,
$$

where $I_{k}: U_{k-1} \rightarrow U_{k}$ is the coarse-to-fine intergrid transfer operator and $A_{k}$ is the bilinear form on $U_{k}$. However, (1) does not hold for most nonnested multigrid methods. Thus the BPX framework produces some nonoptimal mathematics results, such as the variable $\mathrm{V}$-cycle, nonuniform convergence rate, and multigrid preconditioners, (cf. [11, [37]) though most of these methods provide the optimal order of computation. The question has remained open for a long time whether one can lift this obvious limit, the inequality (1), from the BPX framework.

This question will be answered in this paper. We will extend the BPX framework so that the number of smoothings can play its important role in the $\mathrm{V}$-cycle analysis so that the BPX framework can provide a uniform convergence rate without the nearly nested bound (1). We will then apply the extended BPX framework to show the uniform convergence rates of several common nonnested multigrid methods. Some of them were proven previously for two-level and W-cycle iterations only.

So far, we still require the full elliptic regularity assumption in our applications of the BPX framework. Brenner recently gave a proof in [17] for the nonconforming V-cycle multigrid method applied to the second-order elliptic problem, under a lower regularity requirement. It is a better result. In addition, the analysis 17 can be extended to some other nonnested multigrid methods; cf. 44. However, it is not straightforward to apply Brenner's analysis to different cases in general, due to its lengthy analysis and its long list of approximation properties and inverse estimates. For example, the standard inverse estimate fails to hold on the combined space of finite element functions on two nonnested grids such as the ones in Figure 1. In contrast to [17, our extended BPX framework is simple in analysis and can be applied to all common nonnested cases.

The outline of this paper is as follows. In Section 2 we recall the V-cycle multigrid method. The convergence analysis is given in Section 3. In Section 4 we provide a proof for the regularity-approximation assumption for various nonnested methods. Some numerical results will be provided in Section 5 to support the theoretical analysis in this paper.

\section{The V-CyCle MUltigRid Method}

For $k \geq 0$, let $U_{k}$ be a sequence of finite-dimensional vector spaces, along with coarse-to-fine intergrid transfer operators $I_{k}: U_{k-1} \rightarrow U_{k}$. Let $A_{k}(\cdot, \cdot)$ and $(\cdot, \cdot)_{k}$ be symmetric positive definite discrete bilinear forms on $U_{k} \times U_{k}$. We solve the following linear system of equations. Given $f \in U_{k}$, find $v \in U_{k}$ satisfying

$$
A_{k}(v, \phi)=(f, \phi)_{k} \quad \forall \phi \in U_{k} .
$$


To define a V-cycle multigrid method for (2), following the notations in [11, we introduce operators $A_{k}: U_{k} \rightarrow U_{k}, P_{k-1}: U_{k} \rightarrow U_{k-1}$ and $P_{k-1}^{0}: U_{k} \rightarrow U_{k-1}$ as:

$$
\begin{aligned}
\left(A_{k} w, \phi\right)_{k} & =A_{k}(w, \phi) & & \forall \phi \in U_{k}, \\
A_{k-1}\left(P_{k-1} w, \phi\right) & =A_{k}\left(w, I_{k} \phi\right) & & \forall \phi \in U_{k-1}, \\
\left(P_{k-1}^{0} w, \phi\right)_{k-1} & =\left(w, I_{k} \phi\right)_{k} & & \forall \phi \in U_{k-1} .
\end{aligned}
$$

We also introduce linear smoothing operators $R_{k}: U_{k} \rightarrow U_{k}$, along with the adjoint operators $R_{k}^{t}$ with respect to the inner product $(\cdot, \cdot)_{k}$. We define

$$
R_{k}^{(l)}= \begin{cases}R_{k} & \text { if } l \text { is odd } \\ R_{k}^{t} & \text { if } l \text { is even. }\end{cases}
$$

Now we define the standard (symmetric) V-cycle multigrid method [11].

Let $m$ be a positive integer, the number of fine-level smoothings. The multigrid operator $B_{k}: U_{k} \rightarrow U_{k}$ is defined by induction as follows. Set $B_{0}=A_{0}^{-1}$. Assume that $B_{k-1}$ has been defined, and define $B_{k} g \in U_{k}$ for $g \in U_{k}$ as follows.

(i) Set $x^{0}=0$.

(ii) Define $x^{l}$ for $l=1,2, \ldots, m$ by

$$
x^{l}=x^{l-1}+R_{k}^{(l+m)}\left(g-A_{k} x^{l-1}\right) .
$$

(iii) Define $y^{m}=x^{m}+I_{k} q^{1}$, where $q^{1}$ is defined by

$$
q^{1}=B_{k-1} P_{k-1}^{0}\left(g-A_{k} x^{m}\right) .
$$

(iv) Define $y^{l}$ for $l=m+1, m+2, \ldots, 2 m$ by

$$
y^{l}=y^{l-1}+R_{k}^{(l+m)}\left(g-A_{k} y^{l-1}\right) .
$$

(v) Set $B_{k} g=y^{2 m}$.

\section{The CONVERgEnCE ANALYSiS}

To analyze the convergence, we set $J_{k}=I-R_{k} A_{k}$ and $J_{k}^{*}=I-R_{k}^{t} A_{k}$, where $J_{k}^{*}$ denotes the adjoint of $J_{k}$ with respect to $A_{k}(\cdot, \cdot)$ and $I$ is the identity operator. Set

$$
\tilde{J}_{k}^{(m)}= \begin{cases}\left(J_{k}^{*} J_{k}\right)^{m / 2} & \text { if } m \text { is even, } \\ \left(J_{k}^{*} J_{k}\right)^{(m-1) / 2} J_{k}^{*} & \text { if } m \text { is odd. }\end{cases}
$$

We then have the following recursive relation among the multigrid operators (cf. 11])

$$
I-B_{k} A_{k}=\left(\tilde{J}_{k}^{(m)}\right)^{*}\left[\left(I-I_{k} P_{k-1}\right)+I_{k}\left(I-B_{k-1} A_{k-1}\right) P_{k-1}\right] \tilde{J}_{k}^{(m)} .
$$

We make two standard hypotheses (cf. [11) as follows:

(C1) Regularity-approximation assumption

$$
\left|A_{k}\left(\left(I-I_{k} P_{k-1}\right) u, u\right)\right| \leq C_{1} \frac{\left\|A_{k} u\right\|_{k}^{2}}{\lambda_{k}} \quad \forall u \in U_{k},
$$

where $\lambda_{k}$ is the largest eigenvalue of $A_{k}, C_{1}$ is independent of $k$, and $\|\cdot\|_{k}$ is the norm corresponding to $(\cdot, \cdot)_{k}$. In addition, we require that (see remarks below)

$$
\left(A_{k}\left(\left(I-I_{k} P_{k-1}\right) u,\left(I-I_{k} P_{k-1}\right) u\right)\right)^{1 / 2} \leq C_{Q}\left(A_{k}(u, u)\right)^{1 / 2} \quad \forall u \in U_{k},
$$

where $C_{Q}$ is independent of $k$. 
(C2)

$$
\frac{\|u\|_{k}^{2}}{\lambda_{k}} \leq C_{R}\left(\tilde{R}_{k} u, u\right)_{k} \quad \forall u \in U_{k},
$$

where $\tilde{R}_{k}=\left(I-J_{k}^{*} J_{k}\right) A_{k}^{-1}$ and $C_{R}$ is independent of $k$.

Remark 3.1. The smoothing hypothesis (C2) can be easily verified for point, line, and block versions of the Jacobi and Gauss-Seidel iterations (cf. 8], for example). The verification of the regularity-approximation hypothesis $(\mathbf{C 1})$ will be carried out in the next section for many examples. The requirement (3) can be verified easily for all practical cases. Inequality (3) is a simple corollary (cf. [43] for example) of the stability estimate (see (10)

$$
A_{k}\left(I_{k} u, I_{k} u\right) \leq C A_{k-1}(u, u) \quad \forall u \in U_{k-1} .
$$

Theorem 3.1. Assume that (C1) and (C2) hold. Then, for all $k \geq 0$,

$$
\left|A_{k}\left(\left(I-B_{k} A_{k}\right) u, u\right)\right| \leq \delta A_{k}(u, u) \quad \forall u \in U_{k},
$$

where

$$
\delta=\frac{C_{1} C_{R}}{m-C_{1} C_{R}}
$$

with $m>2 C_{1} C_{R}$.

Proof. The method here is motivated by [1], [7, reasoning by mathematical induction. For $k=0$, we have a zero on the left-hand side of (5), and (5) holds. It is assumed that (5) and (6) hold for $k-1$. In what follows, we show that (5) and (6) hold for $k$ too.

In view of $(\mathbf{C 1})$, we have

$$
\left|A_{k}\left(\left(I-I_{k} P_{k-1}\right) \tilde{J}_{k}^{(m)} u, \tilde{J}_{k}^{(m)} u\right)\right| \leq C_{1} \frac{\left\|A_{k} \tilde{J}_{k}^{(m)} u\right\|_{k}^{2}}{\lambda_{k}} .
$$

Define

$$
\bar{J}_{k}= \begin{cases}J_{k}^{*} J_{k} & \text { if } m \text { is even, } \\ J_{k} J_{k}^{*} & \text { if } m \text { is odd. }\end{cases}
$$

By (C2) we have

$$
\frac{\left\|A_{k} \tilde{J}_{k}^{(m)} u\right\|_{k}^{2}}{\lambda_{k}} \leq C_{R} A_{k}\left(\left(I-\bar{J}_{k}\right) \bar{J}_{k}^{m} u, u\right) .
$$

Since the spectrum of $\bar{J}_{k}$ is in $[0,1]$, as shown in [11, [7, we have

(9) $A_{k}\left(\left(I-\bar{J}_{k}\right) \bar{J}_{k}^{m} u, u\right) \leq \frac{1}{m} \sum_{i=0}^{m-1} A_{k}\left(\left(I-\bar{J}_{k}\right) \bar{J}_{k}^{i} u, u\right)=\frac{1}{m}\left\{A_{k}(u, u)-A_{k}\left(\bar{J}_{k}^{m} u, u\right)\right\}$.

Note that $A_{k}\left(\bar{J}_{k}^{m} u, u\right)=A_{k}\left(\tilde{J}_{k}^{(m)} u, \tilde{J}_{k}^{(m)} u\right)$. We then get, by (7)-(9), that

$$
\left|A_{k}\left(\left(I-I_{k} P_{k-1}\right) \tilde{J}_{k}^{(m)} u, \tilde{J}_{k}^{(m)} u\right)\right| \leq \frac{C_{1} C_{R}}{m}\left\{A_{k}(u, u)-A_{k}\left(\tilde{J}_{k}^{(m)} u, \tilde{J}_{k}^{(m)} u\right)\right\} .
$$

Set

$$
t:=\frac{A_{k}\left(\tilde{J}_{k}^{(m)} u, \tilde{J}_{k}^{(m)} u\right)}{A_{k}(u, u)} \quad \forall u \neq 0, u \in U_{k}
$$


or $t:=0$ for $u=0$. Clearly, $t \in[0,1]$. We now rewrite (10) as

$$
\left|A_{k}\left(\left(I-I_{k} P_{k-1}\right) \tilde{J}_{k}^{(m)} u, \tilde{J}_{k}^{(m)} u\right)\right| \leq \frac{C_{1} C_{R}(1-t)}{m} A_{k}(u, u) .
$$

On the other hand, from the Cauchy-Schwarz inequality and (3) we have

$$
\begin{aligned}
& \left|A_{k}\left(\left(I-I_{k} P_{k-1}\right) \tilde{J}_{k}^{(m)} u, \tilde{J}_{k}^{(m)} u\right)\right| \\
\leq & \left\{A_{k}\left(\left(I-I_{k} P_{k-1}\right) \tilde{J}_{k}^{(m)} u,\left(I-I_{k} P_{k-1}\right) \tilde{J}_{k}^{(m)} u\right)\right\}^{\frac{1}{2}}\left\{A_{k}\left(\tilde{J}_{k}^{(m)} u, \tilde{J}_{k}^{(m)} u\right)\right\}^{\frac{1}{2}} \\
\leq & C_{Q} A_{k}\left(\tilde{J}_{k}^{(m)} u, \tilde{J}_{k}^{(m)} u\right)=C_{Q} t A_{k}(u, u) .
\end{aligned}
$$

Combining (12) and (13), we get

$$
\left|A_{k}\left(\left(I-I_{k} P_{k-1}\right) \tilde{J}_{k}^{(m)} u, \tilde{J}_{k}^{(m)} u\right)\right| \leq \min \left\{C_{Q} t, \frac{C_{1} C_{R}}{m}(1-t)\right\} A_{k}(u, u) .
$$

By the relation

$$
A_{k-1}\left(P_{k-1} \tilde{J}_{k}^{(m)} u, P_{k-1} \tilde{J}_{k}^{(m)} u\right)=A_{k}\left(\tilde{J}_{k}^{(m)} u, \tilde{J}_{k}^{(m)} u\right)-A_{k}\left(\tilde{J}_{k}^{(m)} u,\left(I-I_{k} P_{k-1}\right) \tilde{J}_{k}^{(m)} u\right),
$$

the induction hypothesis and the symmetry of $A_{k}$, we get

$$
\begin{aligned}
\mid A_{k}( & \left.\left(I-B_{k} A_{k}\right) u, u\right) \mid \\
\leq & \left|A_{k}\left(\left(I-I_{k} P_{k-1}\right) \tilde{J}_{k}^{(m)} u, \tilde{J}_{k}^{(m)} u\right)\right| \\
& +\left|A_{k-1}\left(\left(I-B_{k-1} A_{k-1}\right) P_{k-1} \tilde{J}_{k}^{(m)} u, P_{k-1} \tilde{J}_{k}^{(m)} u\right)\right| \\
\leq & (1+\delta)\left|A_{k}\left(\left(I-I_{k} P_{k-1}\right) \tilde{J}_{k}^{(m)} u, \tilde{J}_{k}^{(m)} u\right)\right|+\delta A_{k}\left(\tilde{J}_{k}^{(m)} u, \tilde{J}_{k}^{(m)} u\right) \\
\leq & (1+\delta) \min \left\{C_{Q} t, \frac{C_{1} C_{R}}{m}(1-t)\right\} A_{k}(u, u)+\delta t A_{k}(u, u) .
\end{aligned}
$$

Now, to show that (5) and (6) for $k$, we only need to verify

$$
(1+\delta) \min \left\{C_{Q} t, \frac{C_{1} C_{R}}{m}(1-t)\right\}+\delta t \leq \frac{C_{1} C_{R}}{m-C_{1} C_{R}} \quad \forall t \in[0,1] .
$$

When $t=0$, the left-hand side of (15) is zero. When $t=1$, (15) is the induction hypothesis. Next, we consider the case of $t \in(0,1)$. To show (15), by the hypothesis (6) on level $k-1$, it suffices to show that

$$
(1+\delta) C_{Q} \min \left(\frac{t}{1-t}, \frac{C_{1} C_{R}}{C_{Q} m}\right) \leq \delta .
$$

We consider two cases. First,

$$
\frac{C_{1} C_{R}}{C_{Q} m+C_{1} C_{R}} \leq t<1,
$$

i.e.,

$$
\frac{t}{1-t} \geq \frac{C_{1} C_{R}}{C_{Q} m}
$$

Thus

$$
\min \left(\frac{t}{1-t}, \frac{C_{1} C_{R}}{C_{Q} m}\right)=\frac{C_{1} C_{R}}{C_{Q} m},
$$

and

$$
(1+\delta) C_{Q} \min \left(\frac{t}{1-t}, \frac{C_{1} C_{R}}{C_{Q} m}\right)=\frac{C_{1} C_{R}}{m-C_{1} C_{R}} .
$$


For the second case,

i.e.,

$$
0<t \leq \frac{C_{1} C_{R}}{C_{Q} m+C_{1} C_{R}}
$$

we have

$$
\frac{t}{1-t} \leq \frac{C_{1} C_{R}}{C_{Q} m}
$$

$$
\min \left(\frac{t}{1-t}, \frac{C_{1} C_{R}}{C_{Q} m}\right)=\frac{t}{1-t},
$$

and

$$
(1+\delta) C_{Q} \min \left(\frac{t}{1-t}, \frac{C_{1} C_{R}}{C_{Q} m}\right)=\frac{m C_{Q}}{m-C_{1} C_{R}} \frac{t}{1-t} \leq \frac{C_{1} C_{R}}{m-C_{1} C_{R}} .
$$

Thus, equation (16) holds for both cases (17) and (18).

Remark 3.2. Note that from (14) we can get

$$
\left|A_{k}\left(\left(I-I_{k} P_{k-1}\right) \tilde{J}_{k}^{(m)} u, \tilde{J}_{k}^{(m)} u\right)\right| \leq \frac{C_{1} C_{R}}{m+\frac{C_{1} C_{R}}{C_{Q}}} A_{k}(u, u),
$$

which indicates that the number of smoothings, $m$, has to be large enough for the convergence rate in the interval $(0,1)$ in general, even for the two-level method.

On the other hand, if $C_{Q}=1$, we get a convergence rate in $(0,1)$ by (19), for the two-level method, for any $m \geq 1$. Note that if

$$
A_{k}\left(I_{k} P_{k-1} v, I_{k} P_{k-1} v\right) \leq 2 A_{k-1}\left(P_{k-1} v, P_{k-1} v\right) \quad \forall v \in U_{k}, \forall k,
$$

then $C_{Q}=1$. In some cases, (20) holds (see [11] and [22]). Note that (20) is a generalization of (1).

Remark 3.3. The key step in our proof is the introduction of a variable $t$ in (11). By it, we extend the BPX framework from the very limited case (10) to the general case (3), or just (4).

\section{Verification of (C1)}

In this section, we provide a proof for the regularity-approximation assumption (C1) in solving the symmetric and positive definite second-order elliptic problems by various nonnested methods.

Let $\Omega$ be a bounded, connected domain in $\mathbb{R}^{n}, n=2$ or 3 , with Lipschitz continuous boundary $\partial \Omega$. We will use the Sobolev space $H^{l}(\Omega), l \geq 0$, with the norm and seminorm $\|\cdot\|_{H^{l}(\Omega)}$ and $|\cdot|_{H^{l}(\Omega)}$. The $L^{2}(\Omega)\left(=H^{0}(\Omega)\right)$ inner product is denoted by $(\cdot, \cdot)_{L^{2}(\Omega)}$.

We set $U=H_{0}^{1}(\Omega)$ and $A(u, v)=\sum_{|\alpha|,|\beta| \leq 1} \int_{\Omega} a_{\alpha \beta}(x) \partial^{\alpha} u \partial^{\beta} v$, where $\alpha, \beta$ are $n$-indexes and $a_{\alpha \beta}(x) \in L^{\infty}(\Omega)$. Let $\mathcal{J}_{k}, k \geq 0$ denote a sequence of shape-regular triangulations of $\Omega$, with the mesh-size $h_{k}$; cf. [19. On $\mathcal{J}_{k}$, let $U_{k}$ be a finitedimensional space and $A_{k}(\cdot, \cdot)$ a discrete form on $U_{k} \times U_{k}$.

We first list some general hypotheses.

H1) We require that $A(\cdot, \cdot)$ is symmetric and positive definite, and that for any given $f \in L^{2}(\Omega)$ there is a unique solution $u \in U$ such that

$$
A(u, v)=(f, v)_{L^{2}(\Omega)} \quad \forall v \in U
$$


and that $u \in H^{2}(\Omega)$ satisfies

$$
\|u\|_{H^{2}(\Omega)} \leq C\|f\|_{L^{2}(\Omega)}
$$

H2) For all $k$, we require that $A_{k}(\cdot, \cdot)$ is a symmetric, positive definite and bounded bilinear form. We set

$$
\|v\|_{1, k}:=\sqrt{A_{k}(v, v)} \quad \forall v \in U_{k}, \forall k .
$$

H3) Let $\Pi_{j} u \in U_{j}$ denote the standard interpolant to $u \in H^{2}(\Omega)$. For all $k$, we require that

$$
\left\|u-\Pi_{j} u\right\|_{L^{2}(\Omega)}+h_{k}\left\|u-\Pi_{j} u\right\|_{1, j} \leq C h_{k}^{2}\|u\|_{H^{2}(\Omega)}, \quad j=k-1, k .
$$

H4) Let $I_{k}: U_{k-1} \rightarrow U_{k}$ denote the coarse-to-fine intergrid transfer operator. For all $k$, we require that

$$
\left\|I_{k} v\right\|_{L^{2}(\Omega)} \leq C\|v\|_{L^{2}(\Omega)} \quad \forall v \in U_{k-1} .
$$

H5) For all $k$, we require that

$$
C^{-1}\|v\|_{L^{2}(\Omega)} \leq\|v\|_{k} \leq C\|v\|_{L^{2}(\Omega)} \quad \forall v \in U_{k} .
$$

H6) For all $k$, we require that the following inverse inequality holds,

$$
\|\| v\left\|_{1, k} \leq C h_{k}^{-1}\right\| v \|_{L^{2}(\Omega)} \quad \forall v \in U_{k} .
$$

H7) Let $u_{j} \in U_{j}$ be a finite-element approximation to $u$, the exact solution for a given $f \in L^{2}(\Omega)$, i.e.

$$
A(u, v)=(f, v)_{L^{2}(\Omega)} \quad \forall v \in U, \quad A_{j}\left(u_{j}, v\right)=(f, v)_{L^{2}(\Omega)} \quad \forall v \in U_{j} .
$$

For all $k$, we require that

$$
\left\|u-u_{j}\right\|_{L^{2}(\Omega)}+h_{k}\|\| u-u_{j}\left\|_{1, j} \leq C h_{k}^{2}\right\| f \|_{L^{2}(\Omega)}, \quad j=k-1, k .
$$

Remark 4.1. For $C^{0}$ conforming elements and nonconforming elements such as the Crouzeix-Raviart element, usually H7) results from H1)-H3) (cf. [19], 35] and [18]).

Theorem 4.1. Assume H2), H5) and H6). If the following assumption,

(C1)'

$$
\left\|\left(I-I_{k} P_{k-1}\right) v\right\|_{k} \leq C h_{k}^{2}\left\|A_{k} v\right\|_{k} \quad \forall v \in U_{k}
$$

holds, then (C1) holds.

Proof. By H2), the eigenvalues $\lambda_{k, i}$ and eigenvectors $\psi_{k, i}, 1 \leq i \leq N_{k}$, satisfy

$$
\begin{gathered}
A_{k}\left(\psi_{k, i}, v\right)=\lambda_{k, i}\left(\psi_{k, i}, v\right)_{k} \quad \forall v \in U_{k}, \\
0<\lambda_{k, 1} \leq \lambda_{k, 2} \leq \cdots \leq \lambda_{k, N_{k}}, \\
\left(\psi_{k, i}, \psi_{k, j}\right)_{0, k}=\delta_{i j}, \quad A_{k}\left(\psi_{k, i}, \psi_{k, j}\right)=\lambda_{k, i} \delta_{i j},
\end{gathered}
$$

where $\delta_{i j}$ is the Kronecker symbol. It can be easily seen that

$$
\left\|A_{k}^{1 / 2} w\right\|_{k}^{2}=\left(A_{k} w, w\right)_{k} \leq \lambda_{k, N_{k}}(w, w)_{k} \quad \forall w \in U_{k} .
$$

Set $\lambda_{k}:=\lambda_{k, N_{k}}$. From H5) and H6) we see that

$$
\lambda_{k} \leq C h_{k}^{-2} \text {. }
$$

By (C1)' we can conclude that (C1) holds, since

$$
\left|A_{k}\left(\left(I-I_{k} P_{k-1}\right) v, v\right)\right| \leq\left\|\left(I-I_{k} P_{k-1}\right) v\right\|_{k}\left\|A_{k} v\right\|_{k} \leq C h_{k}^{2}\left\|A_{k} v\right\|_{k}^{2} \leq C_{1} \frac{\left\|A_{k} v\right\|_{k}^{2}}{\lambda_{k}}
$$


and

$$
\begin{aligned}
\left(A_{k}\left(\left(I-I_{k} P_{k-1}\right) v,\left(I-I_{k} P_{k-1}\right) v\right)\right)^{1 / 2} & =\|\|\left(I-I_{k} P_{k-1}\right) v \|_{1, k} \\
& \leq C h_{k}^{-1}\left\|\left(I-I_{k} P_{k-1}\right) v\right\|_{k} \\
& \leq C h_{k}\left\|A_{k} v\right\|_{k}=C h_{k}\left\|A_{k}^{1 / 2} A_{k}^{1 / 2} v\right\|_{k} \\
& \leq C h_{k} \lambda_{k}^{1 / 2}\left(A_{k}(v, v)\right)^{1 / 2} \\
& \leq C_{Q}\left(A_{k}(v, v)\right)^{1 / 2} .
\end{aligned}
$$

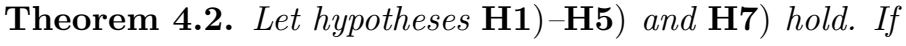

$$
\left\|w_{k}-I_{k} w_{k-1}\right\|_{L^{2}(\Omega)} \leq C h_{k}^{2}\|g\|_{L^{2}(\Omega)}
$$

holds, then (C.1)' holds. Here $w_{j} \in U_{j}$ denotes the finite-element solution on the $j$-th level for a given $g \in L^{2}(\Omega)$; i.e.

$$
A_{j}\left(w_{j}, q\right)=(g, q)_{L^{2}(\Omega)} \quad \forall q \in U_{j} .
$$

Proof. The proof is divided into two steps. In the first step, we show that (21) implies

$$
\left\|w_{k-1}-P_{k-1} w_{k}\right\|_{L^{2}(\Omega)} \leq C h_{k}^{2}\|g\|_{L^{2}(\Omega)} .
$$

To do so, we consider a dual problem: Find $z \in U$ such that

$$
A(z, q)=\left(w_{k-1}-P_{k-1} w_{k}, q\right)_{L^{2}(\Omega)} \quad \forall q \in U .
$$

Denote by $z_{j} \in U_{j}$ the finite element solution to (23); i.e.

$$
A_{j}\left(z_{j}, q\right)=\left(w_{k-1}-P_{k-1} w_{k}, q\right)_{L^{2}(\Omega)} \quad \forall q \in U_{j} .
$$

Applying (21) with a right-hand side function $w_{k-1}-P_{k-1} w_{k} \in L^{2}(\Omega)$, by H7) and the triangle inequality, we have

$$
\begin{aligned}
& \left\|z_{k-1}-I_{k} z_{k-1}\right\|_{L^{2}(\Omega)} \\
& \leq\left\|z_{k-1}-z_{k}\right\|_{L^{2}(\Omega)}+\left\|z_{k}-I_{k} z_{k-1}\right\|_{L^{2}(\Omega)} \\
& \leq\left\|z_{k-1}-z\right\|_{L^{2}(\Omega)}+\left\|z-z_{k}\right\|_{L^{2}(\Omega)}+\left\|z_{k}-I_{k} z_{k-1}\right\|_{L^{2}(\Omega)} \\
& \leq C h_{k}^{2}\left\|w_{k-1}-P_{k-1} w_{k}\right\|_{L^{2}(\Omega)} .
\end{aligned}
$$

Therefore,

$$
\begin{aligned}
\left\|w_{k-1}-P_{k-1} w_{k}\right\|_{L^{2}(\Omega)}^{2} & =A_{k-1}\left(z_{k-1}, w_{k-1}-P_{k-1} w_{k}\right) \\
& =A_{k-1}\left(z_{k-1}, w_{k-1}\right)-A_{k-1}\left(z_{k-1}, P_{k-1} w_{k}\right) \\
& =A_{k-1}\left(z_{k-1}, w_{k-1}\right)-A_{k}\left(I_{k} z_{k-1}, w_{k}\right) \\
& =\left(g, z_{k-1}-I_{k} z_{k-1}\right)_{L^{2}(\Omega)} \\
& \leq C h_{k}^{2}\|g\|_{L^{2}(\Omega)}\left\|w_{k-1}-P_{k-1} w_{k}\right\|_{L^{2}(\Omega)} .
\end{aligned}
$$

It follows that (22) holds.

Now we take the second step, showing (C1)'. To do so, set $E_{k}:=I-I_{k} P_{k-1}$. Again, we consider a dual problem: Find $z \in U$ such that

$$
A(z, q)=\left(E_{k} v, q\right)_{L^{2}(\Omega)} \quad \forall q \in U .
$$

Let $z_{j} \in U_{j}$ be the finite-element solution, approximating $z$; i.e.,

$$
A_{j}\left(z_{j}, q\right)=\left(E_{k} v, q\right)_{L^{2}(\Omega)} \quad \forall q \in U_{j} .
$$

From (21) and (22) we have

$$
\left\|z_{k}-I_{k} z_{k-1}\right\|_{L^{2}(\Omega)}+\left\|z_{k-1}-P_{k-1} z_{k}\right\|_{L^{2}(\Omega)} \leq C h_{k}^{2}\left\|E_{k} v\right\|_{L^{2}(\Omega)} .
$$


Therefore, in view of $\mathbf{H 4}$ ) and $\mathbf{H 5}$ ),

$$
\begin{aligned}
\left\|E_{k} v\right\|_{L^{2}(\Omega)}^{2} & =A_{k}\left(E_{k} v, z_{k}\right) \\
& =A_{k}\left(v, z_{k}\right)-A_{k-1}\left(P_{k-1} v, P_{k-1} z_{k}\right) \\
& =A_{k}\left(v, z_{k}-I_{k} z_{k-1}\right)+A_{k}\left(v, I_{k}\left(z_{k-1}-P_{k-1} z_{k}\right)\right) \\
& \leq\left\|A_{k} v\right\|_{k}\left\|z_{k}-I_{k} z_{k-1}\right\|_{k}+\left\|A_{k} v\right\|_{k}\left\|I_{k}\left(z_{k-1}-P_{k-1} z_{k}\right)\right\|_{k} \\
& \leq C\left\|A_{k} v\right\|_{k}\left\{\left\|z_{k}-I_{k} z_{k-1}\right\|_{L^{2}(\Omega)}+\left\|I_{k}\left(z_{k-1}-P_{k-1} z_{k}\right)\right\|_{L^{2}(\Omega)}\right\} \\
& \leq C\left\|A_{k} v\right\|_{k}\left\{\left\|z_{k}-I_{k} z_{k-1}\right\|_{L^{2}(\Omega)}+\left\|z_{k-1}-P_{k-1} z_{k}\right\|_{L^{2}(\Omega)}\right\} \\
& \leq C h_{k}^{2}\left\|A_{k} v\right\|_{k}\left\|E_{k} v\right\|_{L^{2}(\Omega)},
\end{aligned}
$$

The proof is completed.

Proposition 4.1. Assume $\mathbf{H 1})-\mathbf{H 4}$ ) and $\mathbf{H 7}$ ). If the following estimate holds,

$$
\left\|\Pi_{k} w-I_{k} \Pi_{k-1} w\right\|_{L^{2}(\Omega} \leq C h_{k}^{2}\|w\|_{H^{2}(\Omega)} \quad \forall w \in H^{2}(\Omega),
$$

then (21) holds.

Proof. With a right-hand side function $g \in L^{2}(\Omega)$, let $w \in H^{2}(\Omega)$ be the solution to

$$
A(w, q)=(g, q)_{L^{2}(\Omega)} \quad \forall q \in U .
$$

Let $w_{j} \in U_{j}$ be the finite-element solution to $w$. From $\mathbf{H 7}$ ) we know that

$$
\left\|w-w_{j}\right\|_{L^{2}(\Omega)} \leq C h_{k}^{2}\|g\|_{L^{2}(\Omega)}, \quad j=k-1, k .
$$

Rewriting

$w_{k}-I_{k} w_{k-1}=w_{k}-w+w-\Pi_{k} w+\Pi_{k} w-I_{k} \Pi_{k-1} w+I_{k}\left(\Pi_{k-1} w-w+w-w_{k-1}\right)$, by (26), (24), H3) and H4), we get (21).

Proposition 4.2. Assume $\mathbf{H 1})-\mathbf{H 4}$ ) and $\mathbf{H 7})$. If there exists a finite-dimensional space $\Sigma_{k-1} \subseteq U_{k} \cap U_{k-1}$, which has the same order of approximation as that of $U_{k}$ and $U_{k-1}$, such that

$$
I_{k} w \equiv w \quad \forall w \in \Sigma_{k-1},
$$

or, if the following estimate holds,

$$
\left\|z-I_{k} q\right\|_{L^{2}(\Omega)} \leq C\|z-q\|_{L^{2}(\Omega)} \quad \forall z \in U_{k}, \forall q \in U_{k-1},
$$

then (21) holds.

Proof. Inequality (21) trivially results from (28), the triangle inequality and H7).

Let us assume (27). Let $w_{j} \in U_{j}, j=k, k-1$ and $q_{k-1} \in \Sigma_{k-1}$ be the finiteelement solutions to $w$, for $g \in L^{2}(\Omega)$; cf. (25) and (26).

$$
\begin{aligned}
w_{k}-I_{k} w_{k-1} & =w_{k}-q_{k-1}+q_{k-1}-I_{k} w_{k-1} \\
& =w_{k}-w+w-q_{k-1}+I_{k}\left(q_{k-1}-w+w-w_{k-1}\right) .
\end{aligned}
$$

Inequality (21) follows.

Remark 4.2. For $\mathcal{P}_{1}$ and Wilson's nonconforming elements, (27) is obviously true, with $\Sigma_{k-1}$ being the conforming $\mathcal{P}_{1}$ and $\mathcal{Q}_{1}$ elements, respectively; see [13] and [41.

For $C^{0}$ elements with nonnested triangulations, (28) was shown in [42. For other nonnested $C^{0}$ elements such as a bubble-enriching element and a composed element, (28) was shown in [23]. 
For nonconforming elements such as $\mathcal{Q}_{1}^{\text {rot }}$ element and a discretely divergencefree $\mathcal{P}_{1}$ element, (24) was shown in [36], 4] and [14].

For the Mortar element, (C1)' was shown in [25].

Note that for all these nonnested cases there are different assumptions on the triangulations.

To avoid proliferation, here we leave out more detailed description and verification of assumptions (23), (26) and (27), associated with the assumption (20) for (C1)', for various nonnested V-cycle methods. Readers can refer to the cited references for details.

Remark 4.3. To our best knowledge, all the existing intergrid transfer operators satisfy H4); cf. [43, [1], 4, [2, 14, [13, 41] and 23. Other hypotheses H1)H3) and H5)-H7) are often trivial and standard. We therefore do not insist on details here.

\section{Numerical Results}

In our numerical tests, we studied $P_{1}$ linear triangles and $P_{1}$ nonconforming linear elements, where the nodal values are defined at the vertices or the midedge points, respectively. We tested both nested and nonnested, but uniform grids, (see Figure 1), on the unit square domain $\Omega=(0,1)^{2}$.

The bilinear form is the semi- $H^{1}$ product $A_{k}(u, v)=\int_{\Omega} u_{x} v_{x}+u_{y} v_{y}$. The discrete $L^{2}$ inner product is $(u, v)_{k}=h^{2} \sum u_{i} v_{i}$ where the summation is over all nodal points and $h$ is the grid size.

In Table 1 below, we listed the constants computed numerically for the $P_{1}$ conforming elements on nonnested grids, where $C_{1}$ and $C_{Q}$ are used in the regularityapproximation assumption (C.1), the constant $C_{R}$ is from the smoothing hypothesis (C.2), $\delta_{k}$ (less than the theoretic constant $\delta$ in (5)) is the error reduction factor of the V-cycle nonnested multigrid method, and $\delta_{k}^{\prime}$ is the two-level error reduction factor. Here we solve the coarse-level correction problem exactly in the two-level multigrid method and $\delta_{k}^{\prime}$ is the the spectral radius of such a multigrid operator:

$$
\delta_{k}^{\prime}=\rho\left(\left(\tilde{J}_{k}^{(m)}\right)^{*}\left(I-I_{k} P_{k-1}\right) \tilde{J}_{k}^{(m)}\right) .
$$

We note in particular that all constants are computed by Matlab as they are the maximum or the minimum of certain eigenvalues. Here the number of the smoothing parameter $m$ is set to 8 and the Richardson iteration is used for the presmoothing and the postsmoothing. The same constants for the $P_{1}$ nonconforming elements on nonnested grids are listed in Table 2. Because of the nonnested grids, the intergrid transfer operator $I_{k}$ is simply the nodal value interpolation operator $\Pi_{k}$ as all finelevel nodal points are in the interior of some coarse-level triangles. This avoids
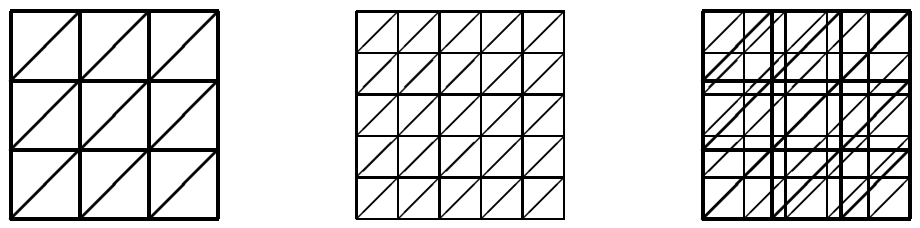

Figure 1. Nonnested grids. $(h=1 / 3$ and $h=1 / 5)$ 
TABLE 1. Constants for $P_{1}$ conforming elements on nonnested grids.

\begin{tabular}{|c|c|c|c|c|c|c|}
\hline level $k$ & grid & $C_{1}$ & $C_{Q}$ & $C_{R}$ & $\delta_{k}$ & $\delta_{k}^{\prime}$ \\
\hline 2 & $3 \times 3$ & 1.7676 & 5.3645 & 1.0000 & 0.0008 & 0.0000 \\
\hline 3 & $5 \times 5$ & 3.3794 & 7.0620 & 1.0000 & 0.0470 & 0.0468 \\
\hline 4 & $9 \times 9$ & 4.8324 & 7.7069 & 1.0000 & 0.0923 & 0.0787 \\
\hline 5 & $17 \times 17$ & 6.0642 & 7.9184 & 1.0000 & 0.1289 & 0.1132 \\
\hline 6 & $33 \times 33$ & 6.9498 & 7.9785 & 1.0000 & 0.1554 & 0.1358 \\
\hline 7 & $65 \times 65$ & 7.5868 & 7.9945 & 1.0000 & 0.1705 & 0.1485 \\
\hline
\end{tabular}

TABle 2. Constants for $P_{1}$ nonconforming elements on nonnested grids.

\begin{tabular}{|c|c|c|c|c|c|c|}
\hline level $k$ & grid & $C_{1}$ & $C_{Q}$ & $C_{R}$ & $\delta_{k}$ & $\delta_{k}^{\prime}$ \\
\hline 2 & $3 \times 3$ & 10.9296 & 31.6111 & 1.0000 & 0.0965 & 0.0261 \\
\hline 3 & $5 \times 5$ & 10.5700 & 26.3688 & 1.0000 & 0.0751 & 0.0741 \\
\hline 4 & $9 \times 9$ & 17.0298 & 38.4648 & 1.0000 & 0.1539 & 0.1521 \\
\hline 5 & $17 \times 17$ & 20.8358 & 44.6080 & 1.0000 & 0.2421 & 0.2051 \\
\hline 6 & $33 \times 33$ & 23.3766 & 52.3772 & 1.0000 & 0.2862 & 0.2422 \\
\hline
\end{tabular}

the trouble of defining nodal (midedge) values of $I_{k} v_{k-1}$, which is done usually by averaging the values of $v_{k-1}$ at nearby nodes; cf. 4, [13, 14, 20, and 22.

Comparing the data in Tables 1 and 2, we can see the constants $C_{1}$ and $C_{Q}$ are much worse for the nonconforming $P_{1}$ elements. However, the V-cycle and the twolevel convergence rates $\delta_{k}$ and $\delta_{k}^{\prime}$ do not differ much between the conforming and nonconforming elements. We note that here the grid size ratio of the fine-to-coarse levels is more than $1 / 2$ - better than that in the nested multigrid method. So the nonnested multigrid convergence rate is better than that of the standard nested multigrid (shown in Table 3).

For Table 3, we have nested grids. We note that because we used one-sided value interpolation operator (since the fine-level midedge points are no longer inside coarse-level triangles) as the intergrid transfer operator, instead of some averaging operators (cf. [13]), the rates of the nonconforming multigrid method (listed in the last two columns) are much worse than that of the conforming method (listed in the middle two columns in Table 3.) Otherwise, the difference in rates should be small, as shown in the nonnested cases (listed in Tables 1 and 21). We further remark that, due to the perturbation to the subspace $A_{k}$-projection, the two-level nonconforming multigrid method is worse than its $\mathrm{V}$-cycle multigrid version in terms of the rate of convergence. In other words, a more accurate coarse-level correction would produce a bigger error to the high-frequency components of the iterative solution on the finer grid. Therefore, if the fine-level smoothing number is not high enough, the multigrid iteration may even diverge. This phenomenon shows up clearly in the last numerical example in this paper.

In Figure 2 we plot the estimated rate $\delta_{k}$ of the V-cycle multigrid method in (6) and the estimated rate in (19) for the two-level multigrid method, against the actual (computed) rates. Here the estimates $\frac{C_{1} C_{R}}{m-C_{1} C_{R}}$ and $\frac{C_{1} C_{R}}{m+C_{1} C_{R} / C_{Q}}$ are both for $P_{1}$ conforming elements, and computed by numerical data $C_{1}$ and $C_{Q}$. The grid level is $k=5$ in Figure 2 , 
TABLE 3. The convergence rate for nested-grid $P_{1}$ conforming and nonconforming elements.

\begin{tabular}{|c|c||c|c||c|c|}
\hline level $k$ & grid & $\delta_{k}(\mathrm{c})$ & $\delta_{k}^{\prime}(\mathrm{c})$ & $\delta_{k}(\mathrm{nc})$ & $\delta_{k}^{\prime}(\mathrm{nc})$ \\
\hline 2 & $4 \times 4$ & 0.0844 & 0.0186 & 0.3211 & 0.1088 \\
\hline 3 & $8 \times 8$ & 0.1619 & 0.1332 & 0.3989 & 0.3775 \\
\hline 4 & $16 \times 16$ & 0.2143 & 0.1663 & 0.4091 & 0.4469 \\
\hline 5 & $32 \times 32$ & 0.2404 & 0.1791 & 0.4359 & 0.4678 \\
\hline
\end{tabular}

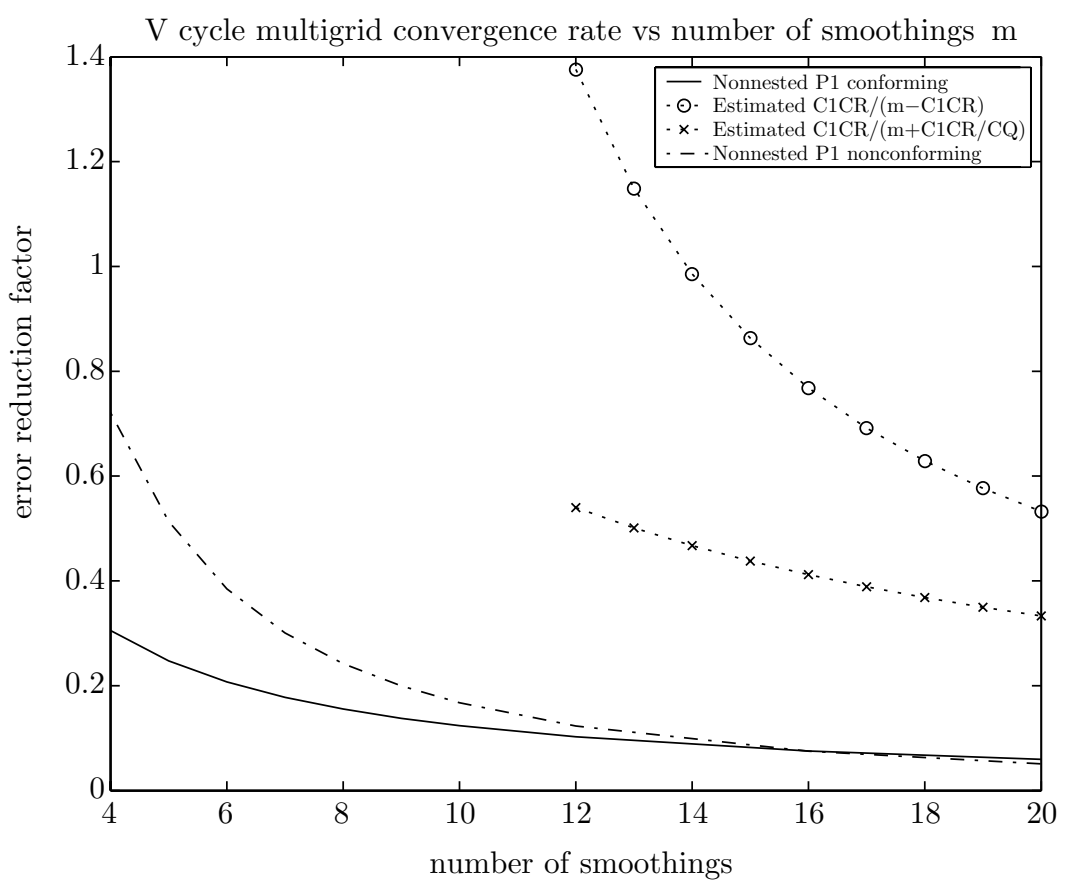

Figure 2. The convergence rate of the V-cycle multigrid

Finally, we would show a counterexample where the number of smoothings $m$ must be sufficiently large, larger than one, depending on a parameter $\sigma$ in the mesh perturbation, in order for the $\mathrm{V}$-cycle nonnested multigrid methods to converge. In the example, we use the cubic Lagrange element to solve the Poisson equation with a homogeneous boundary condition. The domain is the unit square. On the first level, we have only two triangles. We then use the multigrid refinement to generate the higher level meshes. On the fifth level, we perturb the mesh by moving all internal nodes by the mapping $(x, y) /(r / 1.5)^{\sigma}$. In Figure 3. the fine grid is plotted by solid lines and all the coarse grids are plotted (overlapped) by dash lines.

In Table 4. we list the number of V-cycle iterations needed for the $P_{3}$-finite element iterative solution to reach its approximation accuracy. For the nested case, i.e., $\sigma=0$ on the finest level, one smoothing is enough to make $\mathrm{V}$-cycle iteration converge, as predicted by the standard multigrid theory. However, when the meshes are perturbed as shown in Figure 3, one smoothing is not enough for the nonnested V-cycle iteration to converge. As shown in Table 4 the number of smoothings $m$ 


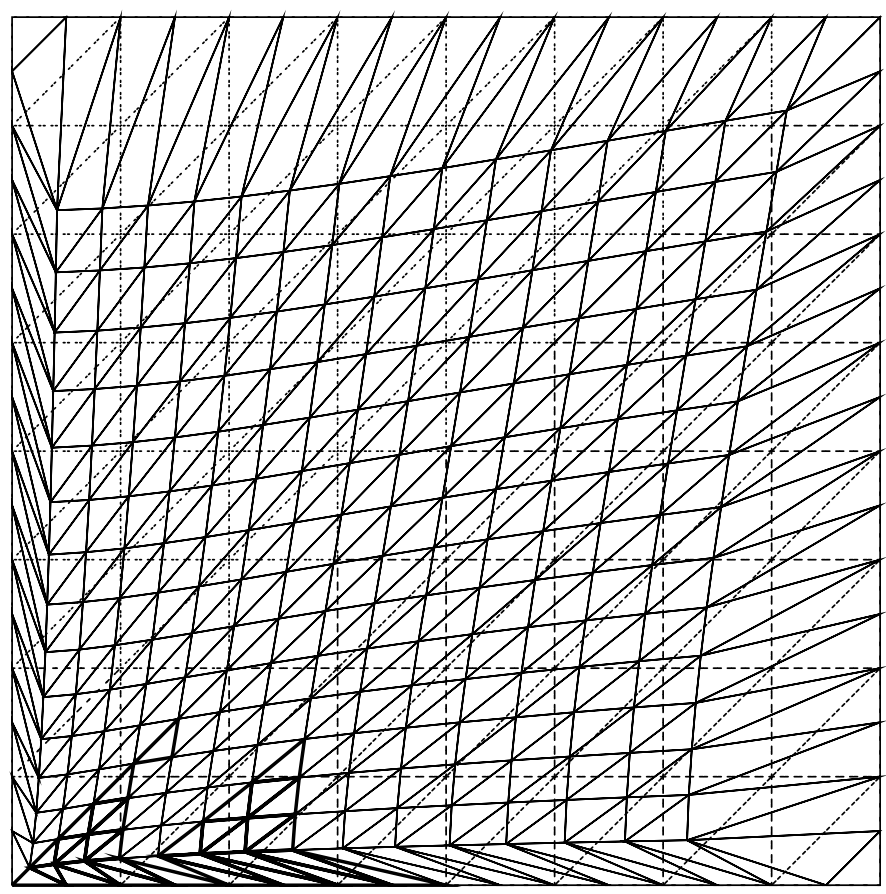

Figure 3. The fifth grid is perturbed by $(x, y) /(r / 1.5)^{0.4}$.

must be larger than 4 (when $\sigma=0.36$ ) or 7 (when $\sigma=0.4$ ), respectively. Otherwise the $\mathrm{V}$-cycle iterations would diverge. From Table 4 it seems that the when $m$ is large, the converge rates of nested and nonnested V-cycles differ very little. We remark that the reduction rate $\delta_{5}$ listed in Table 4 does not decrease monotonically when $m$ increases. This is caused by the way we average the error reduction factors by the number of V-cycles:

$$
\delta_{5}=\frac{1}{n_{v}} \sum_{i=1}^{n_{v}} \frac{\left|u_{h}-u_{i}\right|_{H^{1}}}{\left|u_{h}-u_{i-1}\right|_{H^{1}}},
$$

where $n_{v}$ is the number of V-cycles.

In Figure 4, we plot the iterative error before doing a nonnested coarse-level correction and after doing such a correction. In the nonnested coarse-level correction, the low-frequency components of the iterative error are usually reduced well. However, due to nonnestedness, some high-frequency errors would be amplified. This can be seen by comparing the two graphs in Figure 4. Therefore, the fine-level smoothing has to be performed enough times in order for the nonnested multigrid method to converge.

We make a final remark on selecting the counterexample. The difficulty here arises when we use the multigrid refinement to generate meaningful, or likely practical, grids. With reasonable perturbations of the grids, we could not find a case where the $P 1$ multigrid V-cycle diverges. After numerous successful tries, we turned to $P 2, P 3$ and high-order elements where one fine-level smoothing is not powerful enough to smooth out the non- $a(\cdot, \cdot)$-projection component of the coarse-level correction. 
TABLE 4. The number of V-cycles and the error reduction rate for for nonnested $P_{3}$ elements.

\begin{tabular}{|r||cc||cc||cc|}
\hline$m$ & \# V-cycle, & $\delta_{5}(\sigma=0)$ & \# V-cycle, & $\delta_{5}(\sigma=.36)$ & \# V-cycle, & $\delta_{5}(\sigma=.4)$ \\
\hline 1 & 72 & 0.9035 & $\infty$ & 1.0266 & $\infty$ & 1.4101 \\
2 & 36 & 0.8188 & $\infty$ & 1.2698 & $\infty$ & 1.7989 \\
3 & 24 & 0.7481 & $\infty$ & 1.1905 & $\infty$ & 1.6841 \\
4 & 18 & 0.6900 & $\infty$ & 1.0396 & $\infty$ & 1.4963 \\
5 & 14 & 0.6215 & $>40$ & 0.9304 & $\infty$ & 1.3195 \\
6 & 12 & 0.5848 & $>40$ & 0.8708 & $\infty$ & 1.1638 \\
7 & 10 & 0.5247 & 13 & 0.6203 & $\infty$ & 1.0237 \\
8 & 8 & 0.4374 & 11 & 0.5767 & $>40$ & 0.8833 \\
9 & 7 & 0.3936 & 9 & 0.5107 & $>40$ & 0.8920 \\
10 & 6 & 0.3358 & 8 & 0.4733 & $>40$ & 0.9015 \\
11 & 6 & 0.3651 & 7 & 0.4251 & 10 & 0.5803 \\
12 & 5 & 0.2767 & 7 & 0.4206 & 9 & 0.5615 \\
13 & 5 & 0.3026 & 6 & 0.3673 & 7 & 0.4503 \\
14 & 5 & 0.2821 & 6 & 0.3839 & 6 & 0.4033 \\
15 & 4 & 0.2141 & 6 & 0.4006 & 5 & 0.2900 \\
16 & 4 & 0.2315 & 5 & 0.2917 & 5 & 0.2966 \\
17 & 4 & 0.2479 & 5 & 0.3013 & 5 & 0.3078 \\
18 & 4 & 0.2699 & 5 & 0.3160 & 5 & 0.3148 \\
19 & 4 & 0.2673 & 5 & 0.3341 & 5 & 0.3347 \\
20 & 4 & 0.2690 & 5 & 0.3386 & 5 & 0.3743 \\
21 & 4 & 0.2800 & 5 & 0.3608 & 4 & 0.2111 \\
22 & 4 & 0.3340 & 5 & 0.3729 & 4 & 0.2143 \\
23 & 3 & 0.1602 & 4 & 0.2117 & 4 & 0.2168 \\
24 & 3 & 0.1645 & 4 & 0.2120 & 4 & 0.2217 \\
25 & 3 & 0.1691 & 4 & 0.2159 & 4 & 0.2206 \\
\hline
\end{tabular}
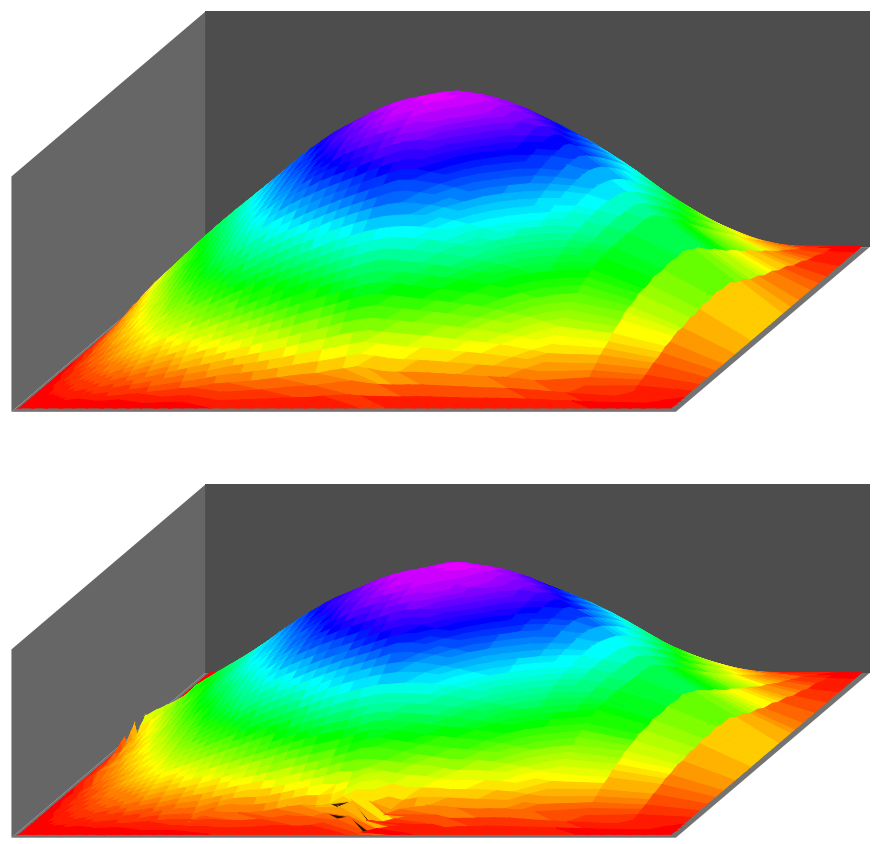

FiguRE 4. The iterative error before and after doing a coarse-level correction. 


\section{REFERENCES}

[1] R.E. Bank and T. Dupont, An optimal order process for solving finite element equations, Math. Comp., 36(1981), 35-51. MR0595040 (82b:65113)

[2] D. Braess, M. Dryja and W. Hackbusch, A multigrid method for nonconforming FE-discretizations, with application to no-matching grids, Computing, 63(1999), 1-25. MR1702163 (2000h:65048)

[3] D. Braess and W. Hackbusch, A new convergence proof for the multigrid method including the V-cycle, SIAM J. Numer. Anal., 20(1983), 967-975. MR0714691(85h:65233)

[4] D. Braess and R. Verfürth, Multigrid methods for nonconforming finite element methods, SIAM J. Numer. Anal., 27(1990), 979-986. MR.1051117 (91j:65164)

[5] J. H. Bramble, Multigrid Methods, Pitman Research Notes in Mathematics, V. 294, John Wiley and Sons, 1993. MR 1247694 (95b:65002)

[6] J. H. Bramble, D.Y. Kwak and J. E. Pasciak, Uniform convergence of multigrid V-cycle iterations for indefinite and nonsymmetric problems, SIAM J. Numer. Anal., 31(1994), 17461763. MR1302683 (95i:65170)

[7] J. H. Bramble and J.E. Pasciak, New convergence estimates for multigrid algorithms, Math. Comp., 49 (1987), 311-329. MR0906174 (89b:65234)

[8] J. H. Bramble and J. E. Pasciak, The analysis of smoothers for multigrid algorithms, Math. Comp., 58(1992) 453-470. MR1122058 (92f:65146)

[9] J. H. Bramble and J. E. Pasciak, New estimates for multilevel algorithms including the Vcycle, Math. Comp., 60(1993) 447-471. MR.1176705 (94a:65064)

[10] J. H. Bramble and J. E. Pasicak, Uniform convergence estimates for multigrid V-cycle algorithms with less than full elliptic regularity, in: Domain Decomposition methods in science andEngineering, A. Quarteroni, J. Periaux, Y. A. Kuznetsov and O. B. Widlund, Eds, Contemporary Mathematics Series 157 Am. Math. Soc. (1994) 17-26. MR1262601 (95f:65202)

[11] J. H. Bramble, J. E. Pasciak and J. Xu, The analysis of multigrid algorithms with nonnested spaces or noninherited quadratic forms, Math. Comp., 56(1991), 1-34. MR1052086 (91h:65159)

[12] J. H. Bramble and X. Zhang, Uniform convergence of the multigrid V-cycle for an anisotropic problem, Math. Comp., 70(2001), 979-986. MR.1709148 (2001g:65134)

[13] S. C. Brenner, An optimal-order multigrid method for $\mathcal{P}_{1}$ nonconforming finite elements, Math. Comp., 52(1989), 1-15. MR0946598 (89f:65119)

[14] S. C. Brenner, A nonconforming multigrid method for the stationary Stokes equations, Math. Comp., 55(1990), 411-437. MR1035927(91d:65167)

[15] S. C. Brenner, Convergence of nonconforming multigrid methods without full elliptic regularity, Math. Comp., 68(1999), 25-53. MR1620215 (99c:65229)

[16] S. C. Brenner, Convergence of the multigrid V-cycle algorithms for second order boundary value problems without full elliptic regularity, Math. Comp., 71 (2002), 507-525. MR:1885612 (2003b:65132)

[17] S. C. Brenner, Convergence of nonconforming V-cycle and F-cycle multigrid algorithms for second order elliptic boundary value problems, Math. Comp., 73 (2004), 1041-1066. MR2047077 (2005f:65167)

[18] S. C. Brenner and L. R. Scott, The Mathematical Theory of Finite Element Methods, Springer-Verlag, New-York, (1996). MR.1278258 (95f:65001)

[19] P. G. Ciarlet, The Finite Element Method for Elliptic Problems, North-Holland, Amsterdam, (1978). MR.0520174 (58:25001)

[20] Z. Chen, On the convergence of Galerkin-multigrid methods for nonconforming finite elements, East-West J. Numer. Math., 7(1999), 79-107. MR1699247 (2000e:65116)

[21] Z. Chen, On the convergence of nonnested multigrid methods with nested spaces on coarse grids, Numer. Methods PDE, 16(2000), 265-284. MR1752413(2001c:65154)

[22] Z. Chen and P. Oswald, Multigrid and multilevel methods for nonconforming rotated Q1 elements, Math. Comp., 67(1998), 667-693. MR1451319 (98g:65118)

[23] Q. Deng and X. Feng, Multigrid methods for the generalized Stokes equations based on mixed finite element methods, J. Comp. Math., 20(2002), 129-152. MR.1884415 (2002k:76084)

[24] C.I. Goldstein, Multigrid analysis of finite element methods with numerical integration, Math. Comp., 56(1991), 409-436. MR1066832(91h:65190)

[25] J. Gopalakrishnan and J. E. Pasciak, Multigrid for the Mortar finite element method, SIAM J. Numer. Anal. 37 (2000) 1029-1052. MR.1749248 (2001b:65124) 
[26] J.Gopalakrishnan and G. Kanschat, Application of unified DG analysis to preconditioning DG methods, in: Bathe, K. J. eds., Computational Fluid and Solid Mechanics 2003, Elsevier, Amsterdam, (2003) 1943-1945. MR2029558 (2004i:74002)

[27] J.Gopalakrishnan and G. Kanschat, A multilevel discontinuous Galerkin method, Numer. Math. 95 (2003) 527-550. MR2012931 (2004m:65179)

[28] W. Hackbusch, Multi-grid Methods and Applications, Springer-Verlag, Berlin, 1985. MR0814495 (87e:65082)

[29] C. Lee, A nonconforming multigrid method using conforming subspaces, in: Proc. Sixth Copper Mountain Conf. on Multigrid Methods, N. Melson et al., eds, NASA Conference Publication 3224, Part 1, Washington, DC, 1993, 317-330.

[30] P. Monk and S. Zhang, Multigrid computation of vector potentials, J. Comp. Appl. Math. 62 (1995), 301-320. MR:1363678 (96h:65149)

[31] S. F. McCormick, ed., Multigrid Methods, SIAM Frontiers in Applied Mathematics, Philadelphia, 1987. MR0972752 (89m:65004)

[32] L. R. Scott and S. Zhang, Higher-dimensional nonnested multigrid methods, Math. Comp. 58 (1992), 457-466. MR1122077 (92g:65133)

[33] V. V. Shaidurov, Multigrid Methods for Finite Elements, Kluwer Academic, the Netherlands, 1989. MR1335921 (97e:65142)

[34] R. Stevevson, An analysis of nonconforming multi-grid methods, leading to an improved method for the Morley element, Math. Comp. 72 (2002), 55-81. MR.1933814 (2003i:65127)

[35] G. Strang and G. J. Fix, An Analysis of the Finite Element Method, Prentice-Hall, Englewood Cliffs, NJ, 1973. MR0443377 (56:1747)

[36] S. Turek, Multigrid techniques for a divergence free finite element discretization, East-West J, Numer. Math., 2(1994), 229-255. MR.1296984 (96c:65195)

[37] J. Wang, Convergence analysis of multigrid algorithms for nonselfadjoint and indefinite elliptic problems, SIAM J. Numer. Anal. 30 (1993), 275-285. MR1202666 (93k:65100)

[38] J. Xu, Theory of Multilevel Methods, PhD thesis, Cornell University, 1989.

[39] X. Xu and J. Chen, Multigrid for the mortar element method for P1 nonconforming element, Numer. Math. 88 (2001), 381-398. MR.1826859 (2002d:65146)

[40] X. Xu , L. Li and W. Chen, A multigrid method for the mortar-type Morley element approximation of a plate bending problem, SIAM J. Numer. Anal. 39 (2002), 1712-1731. MR1885713 (2002m:74050)

[41] X. Yu, Multigrid method of nonconforming Wilson finite element, Math. Numer. Sinica, 14(1993), 346-351. MR.1391414 (97b:65143)

[42] S. Zhang, Optimal order nonnested multigrid methods for solving finite element equations, Math. Comp., 55(1990), I: on quasi-uniform meshes, 23-36; II: on non-quasi-uniform meshes, 439-450. MR1023054 (91g:65268) MR1035947(91g:65269)

[43] S. Zhang and Z. Zhang, Treatments of discontinuity and bubble functions in the multigrid method, Math. Comput., 66(1997), 1055-1072. MR1415804 (97k:65285)

[44] J. Zhao, Convergence of nonconforming V-cycle and F-cycle multigrid methods for the biharmonic problem using the Morley element, IMI Research Report, Math Dept, University of South Caroline, 2003.

Department of Mathematics, National University of Singapore, 2 Science Drive 2 , SINGAPORE 117543

E-mail address: scidhy@nus.edu.sg

College of Mathematics and Computers, Hebei University, 071002, 1 Hezuo Road, Baoding, Hebei, China

E-mail address: gaoshq@amss.ac.cn.

Department of Mathematics, National University of Singapore, 2 Science Drive 2, SINGAPORE 117543

E-mail address: scitance@nus.edu.sg.

Department of Mathematical Sciences, University of Delaware, Newark, Delaware 19716

E-mail address: szhang@udel.edu 